\title{
On the distribution schemes for determining flows through a merge
}

\author{
W. L. Jin and H. M. Zhang
}

April 11, 2002

\begin{abstract}
In this paper, we study various distribution schemes for determining flows through a merge in the supply-demand framework. Upon a thorough analysis of existing schemes, we propose a simple distribution scheme that satisfies the "fairness" condition, in which the flow from an upstream link is proportional to its traffic demand. We demonstrate that this scheme can capture characteristics of a merge, such as characteristic difference between upstream links, capacity of each link, and control of an on-ramp. Moreover, this scheme leads to a merge model that is computationally efficient and easy to calibrate. Wave solutions of a general Riemann problem will be of interest in future studies.
\end{abstract}

Keywords: The kinematic wave traffic flow models, the discrete merge model, traffic demand, traffic supply, the supply-demand method, the distribution scheme, the "fairness" condition.

\section{Introduction}

For developing advanced traffic control strategies (e.g., Chen et al., 1997), dynamic traffic assignment (DTA) algorithms (e.g., Lo, 1999), and other technologies in Advanced Traffic Management Systems (ATMS) and Advanced Traveler Information Systems (ATIS), traffic engineers need the 
assistance of network traffic flow models that can capture system-wide features of traffic dynamics and are computationally efficient for a network of realistic size. The kinematic wave model is a promising candidate for these tasks since it describes traffic phenomena in the aggregate level in terms of expansion and shock waves and as such is highly efficient for simulating traffic dynamics in a large network.

In the seminal kinematic wave model by Lighthill-Whitham (1955) and Richards (1956), a.k.a. the LWR model, how a disturbance in traffic propagates through a link was thoroughly studied. To model traffic dynamics on a network with the kinematic wave theory, however, one needs to carefully study traffic dynamics at a merge, a diverge, or other components of a network. The kinematic wave models of merging traffic have been studied by Daganzo (1995), Holden and Risebro (1995), and Lebacque (1996). In the model by Holden and Risebro, traffic flows through a merge are determined by an optimization problem. However, the physical meaning and the objective function of the optimization problem are not known or supported by observations. On the other hand, the models by Daganzo and Lebacque are based on the definitions of the local traffic supply and demand and can be considered as reasonable extensions of the kinematic wave theory for link traffic flow. In this paper, we will examine the latter models so that they can be better understood, more easily calibrated, and more efficiently applied in simulation.

As we know, the LWR model, in which the evolution of traffic density $\rho(x, t)$, flow-rate $q(x, t)$, and travel speed $v(x, t)$ is studied in space $x$ and time $t$, can be written as a partial differential equation based on the fact of traffic conservation and the adoption of the fundamental diagram. For the purpose of simulation, the LWR model is generally written in a discrete form: a link and a duration of time are partitioned into a number of cells and time steps respectively, and the increment of the number of vehicles in a cell at each time step equals to the difference between the 
inflow into and outflow from that cell during the time step. In the discrete LWR model, to solve the flow through a boundary, which is the inflow into the downstream cell and the outflow from the upstream cell, two equivalent approaches can be used: in the mathematical approach, one solves the Riemann problem at that boundary (Lebacque, 1996; Jin and Zhang, 2000); in the engineering approach, a.k.a. the supply-demand method, the supply of the upstream cell and the demand of the downstream cell are computed first and the flow is their minimum. Here the concepts of supply and demand were first introduced by Daganzo (1995), but using the terms of "sending flow" and "receiving flow" instead; the terms of "supply" and "demand" were first used by Lebacque (1996). The definitions of demand and supply are as follows: the demand of a cell is equal to the flow-rate when the traffic condition is under-critical (i.e., free flow) and the capacity when overcritical (i.e., congested); the supply is equal to the capacity when the traffic condition is under-critical and the flow-rate when overcritical. ${ }^{1}$

For computing flows through a merge, including the outflows from the upstream cells and the inflow into the downstream cell, Daganzo (1995) extended the supply-demand method as follows: the outflow from an upstream cell is smaller than or equal to its demand, the inflow to the downstream cell is smaller than or equal to its supply, and the inflow is equal to the sum of the outflows in order to preserve traffic conservation. In this supply-demand method, the inflow is unique since it is equal to the minimum of the supply and total demand. But the outflow from each upstream cell may not be unique. Thus one has to find a way to distribute to each upstream cell a fraction of the total outflow, which is equal to the inflow. Here we call such a way of determining the distribution fractions the distribution scheme.

Lebacque proposed another extension of the supply-demand method: the supply of the down-

\footnotetext{
${ }^{1}$ From the definitions of demand and supply, we can see that the flow through a boundary is bounded by the capacity.
} 
stream cell is first distributed as a virtual supply to each upstream cell, the outflow from each upstream cell is the minimum of its demand and virtual supply, and the inflow into the downstream cell is equal to the sum of the outflows. Thus the distribution scheme in Lebacque's method is used to determine the fractions of virtual supplies, and is more general since more feasible solutions of flows can be found in this method.

Both Daganzo and Lebacque provided general formulations of the kinematic wave model of merges. Here, we do not intend to extend these formulations. Rather, we are interested in the distribution schemes used in these models since a distribution scheme is the key to uniquely determine flows through a merge. As in possible applications of a merge model one wants to obtain unique flows under given situation, the distribution schemes are worth a thorough examination.

At a first glance, the determination of distribution fractions seems to be complicated since they may be affected by travellers' merging behavior, the geometry of the studied merge, traffic capacities, difference between the upstream cells, traffic conditions, and the control of an onramp. Considering part of these factors, both Daganzo (1995) and Lebacque (1996) provided their suggestions of the distribution scheme: Lebacque suggested that the distribution fraction is proportional to number of lanes in an upstream cell; Daganzo considered that upstream cells bear different priorities and hence introduced parameters for priorities in his distribution scheme. Both suggestions have their limitations: Lebacque's distribution scheme is too simple and fails under certain situations, while Daganzo's scheme becomes very complicated for a merge with three or more upstream links. Therefore, we devote this study to better understanding of the distribution schemes. In this paper, we propose a distribution scheme which is well-defined, computationally efficient, and capable of addressing the aforementioned concerns.

In this paper, we first review the discrete kinematic wave model of merges and discuss different 
formulations of the supply-demand method for computing flows through a merge (Section 2). In Section 3, after discussing existing distribution schemes, we propose a simple distribution scheme, which incorporates the "fairness" condition. In this scheme, the distribution fractions are proportional to traffic demands of upstream cells. This scheme is shown to work well in simulations due to its many merits: it is capable of capturing the characteristic difference between upstream cells (e.g. the speed difference between the upstream freeway and on-ramps); it is easy to calibrate because additional parameters such as priorities do not need to be explicitly introduced; it is efficient in computation. In Section 4, we present an example of two merging flows and demonstrate in numerical simulations that the kinematic wave model with the supply-demand method incorporating the "fairness" condition is well-defined and of first order in convergence. In the conclusion part, we present the supply-demand method for computing flows through a diverge and a general junction for single-commodity traffic flow, and discuss related future research.

\section{The discrete kinematic wave model of merges with the supply- demand method}

In the kinematic wave traffic flow models of a road network with a merge, the LWR model can be used to describe traffic dynamics of each branch, for which flows through the merge can be considered as boundary conditions. Thus, in this section, we first review the discrete LWR model, the definitions of supply and demand, and the supply-demand method for computing flows through link boundaries. AFter reviewing the models of merges under the supply-demand framework by Daganzo (1995) and Lebacque (1996), we then demonstrate the importance of the distribution

scheme. At the end of this section, we will discuss the properties of existing distribution schemes. 


\subsection{The discrete LWR model in the supply-demand framework}

In the LWR model for each branch of a merge, traffic dynamics are governed by a traffic conservation equation,

$$
\rho_{t}+q_{x}=0
$$

and an equilibrium relationship between $\rho$ and $q$, also known as the fundamental diagram,

$$
q=Q(a, \rho)
$$

where $a(x)$ is an inhomogeneity factor, depending on road characteristics, e.g., the number of lanes at $x$. Since $q=\rho v$, we also have a speed-density relation: $v=V(a, \rho) \equiv Q(a, \rho) / \rho$. For vehicular traffic, generally, $v$ is non-increasing and $q$ is concave in $\rho$. Examples of empirical models of speedand flow-density relations can be found in (Newell, 1993; Kerner and Konhäuser, 1994). Related to the fundamental diagram, the following definitions are used in this paper: the maximum flow-rate at $x$ is called the traffic capacity, and the corresponding density is called the critical density; traffic flow is overcritical when its density is higher than the critical density, and under-critical conversely.

From (1) and (2), the LWR model can be written as

$$
\rho_{t}+Q(a, \rho)_{x}=0
$$

where $0 \leq \rho \leq \rho_{j}\left(\rho_{j}\right.$ is the jam density). When $a(x)$ is uniform with respect to location $x$, the LWR model is called homogeneous. Otherwise it is called inhomogeneous. Both the homogeneous and inhomogeneous models are hyperbolic systems of conservation laws. Actually the former, which is a strict hyperbolic conservation law (Lax, 1972), is a special case of the latter, a non-strictly hyperbolic system of conservation laws and a resonant nonlinear system (Isaacson and Temple, 1992). Therefore, the following discussions for the inhomogeneous LWR model are valid for any kind of links. 
With jump initial conditions, the LWR model (3) is solved by shock waves, rarefaction waves, and standing waves. These wave solutions are unique under the so-called "entropy" conditions. However, solutions of the LWR model with general initial and boundary conditions can not be expressed in analytical form, which calls for approximate solutions with numerical methods. One efficient numerical method for solving (3) is due to Godunov (1959). In the Godunov method, the link is partitioned into $N$ cells, a duration of time is discretized into $M$ time steps, and the discretization of space and time satisfies the Courant-Friedrichs-Lewy (Courant et al., 1928) (CFL) condition so that a vehicle is not allowed to cross a cell during a time interval. Assuming that the spacing $\Delta x$ and the time step $\Delta t$ are constant, $\rho_{i}^{m}$ is the average of $\rho$ in the cell $i$ at time step $m, q_{i-1 / 2}^{m+1 / 2}$ and $q_{i+1 / 2}^{m+1 / 2}$ are the inflow into and the outflow from cell $i$ from time step $m$ to $m+1$ respectively, the LWR model (3) for cell $i$ can be approximated with a finite difference equation:

$$
\frac{\rho_{i}^{m+1}-\rho_{i}^{m}}{\Delta t}+\frac{q_{i-1 / 2}^{m+1 / 2}-q_{i+1 / 2}^{m+1 / 2}}{\Delta x}=0 .
$$

In (4), the flow through the link boundary $x_{i-1 / 2}$, i.e., $q_{i-1 / 2}^{m+1 / 2}$, can be computed in two approaches. One is from the wave solutions of the Riemann problem for (3) with the following initial conditions (Jin and Zhang, 2000):

$$
U\left(x=x_{i-1 / 2}, t=t_{m}\right)=\left\{\begin{array}{cc}
U_{i-1}^{m} & x<x_{i-1 / 2} \\
U_{i}^{m} & x>x_{i-1 / 2}
\end{array}\right.
$$

where $U=(a, \rho)$. Another is the supply-demand method (Daganzo, 1995; Lebacque, 1996), in which the flow through a link boundary is the minimum of the traffic demand of its upstream cell and the traffic supply of its downstream cell. The two approaches were shown to be equivalent (Jin and Zhang, 2000). However, the method of solving the Riemann problem and the supply-demand method have different fates for studying traffic dynamics through a merge: there has been no 
formulation of the Riemann problem for merging dynamics in literature, but the supply-demand method has been extended and applied in the discrete kinematic wave models of merges.

In the following, we describe in detail the supply-demand method. Considering the link boundary at $x_{i-1 / 2}$, whose upstream and downstream cells are respectively denoted by cell $i-1$ and cell $i$, supposing that the traffic densities of the two cells are $\rho_{i-1}^{m}$ and $\rho_{i}^{m}$ at time step $m$, Daganzo (1995) and Lebacque (1996) suggested the following supply-demand method for computing $q_{i-1 / 2}^{m+1 / 2}$. First, traffic demand of the cell $i-1$ (called "sending flow" by Daganzo), $D_{i-1}^{m+1 / 2}$, and traffic supply of the cell $i$ (called "receiving flow" by Daganzo), $S_{i}^{m+1 / 2}$, are defined by

$$
\begin{aligned}
& D_{i-1}^{m+1 / 2}= \begin{cases}Q_{i-1}^{m}, & \text { cell } i-1 \text { is under-critical }, \\
Q_{i-1}^{\max }, & \text { otherwise }\end{cases} \\
& S_{i}^{m+1 / 2}= \begin{cases}Q_{i}^{\max }, & \text { cell } i \text { is under-critical, } \\
Q_{i}^{m}, & \text { otherwise }\end{cases}
\end{aligned}
$$

where $Q_{i}^{\max }$ is the capacity of cell $i$, and $Q_{i}^{m}$ the flow-rate of cell $i$ at time step $m$. The demand can be considered as the maximum flow that can be discharged by the cell $i-1$ from time step $m$ to $m+1$; the supply $S_{i}^{m+1 / 2}$ is the maximum flow that can be received by the cell $i$. Thus, the boundary flow satisfies (all superscripts will be suppressed hereafter)

$$
\begin{aligned}
& q_{i-1 / 2} \leq D_{i-1}, \\
& q_{i-1 / 2} \leq S_{i} .
\end{aligned}
$$

Note that (8) admits multiple solutions. To identify the unique boundary flow, an additional "optimality" condition is assumed that the actual boundary flow always reaches its maximum. Hence, the boundary flow can be simply computed by

$$
q_{i-1 / 2}=\min \left\{D_{i-1}, S_{i}\right\}
$$


Here the "optimality" condition can be considered as an entropy condition, which helps to choose a physical solution out of all feasible solutions.

\subsection{The kinematic wave model of merging traffic in the supply-demand frame- work}

In this subsection, we review the kinematic wave model of merging traffic in the supply-demand

method framework. In this type of models, the supply-demand method is used to compute flows through a merge. Without loss of generality, we can consider a merge that connects two upstream cells to one down stream cell. Furthermore, we assume that, at time step $m$, traffic demands of the two upstream cells and the traffic supply of the downstream cell are $D_{1}, D_{2}$, and $S_{d}$ respectively. We denote the outflows from the upstream cells by $q_{1}$ and $q_{2}$ and the inflow into the downstream cell by $q$ from time step $m$ to $m+1$. Then, according to traffic conservation, we have $q=q_{1}+q_{2}$.

The basic assumption in the supply-demand method for computing the flows through a merge is that the flows, $q_{1}, q_{2}$, and $q$, are determined by traffic conditions $D_{1}, D_{2}, S_{d}$, and/or other characteristics of the merge. Another assumption, as in the supply-demand method for computing the flow through a link boundary, is the optimality condition. Two types of optimality conditions have been proposed: one is that the total flow $q$ reaches its maximum, and the other is that both $q_{1}$ and $q_{2}$ reaches their individual maximums. Following the first assumption leads to Daganzo's method (1995), and following the second leads to Lebacque's (1996). 
In Daganzo's supply-demand method, we have the following optimization problem:

$$
\max q=q_{1}+q_{2}
$$

s.t.

$$
\begin{aligned}
& q_{1} \leq D_{1}, \\
& q_{2} \leq D_{2}, \\
& q_{1}+q_{2} \leq S_{d}, \\
& q_{1}, q_{2} \geq 0,
\end{aligned}
$$

from which we can find the total flow,

$$
q=\min \left\{D_{1}+D_{2}, S_{d}\right\}
$$

However, $\left(q_{1}, q_{2}\right)$ may have multiple feasible solutions. This can be shown with Figure 1: when $S_{d} \geq D_{1}+D_{2}$, the solution is unique and at point $Q$; i.e., $\left(q_{1}, q_{2}\right)=\left(D_{1}, D_{2}\right)$; but when $S_{d}<$ $D_{1}+D_{2}$, the solution can be any point on the line segment $A B$. For the latter situation, Daganzo defined two (non-negative) distribution fractions $\alpha_{1}$ and $\alpha_{2}$, which satisfy $\alpha_{1}+\alpha_{2}=1$ and may be related to $D_{1}, D_{2}, S_{d}$, and other characteristics of the merge. Then, the total flow $q$ is distributed by $q_{i}=\alpha_{i} q(i=1,2)$. One example when $S_{d}<D_{1}+D_{2}$ is depicted in the figure, with given fractions $\alpha_{1}$ and $\alpha_{2}$. Figure 1 also shows that $\alpha_{1}$ or $\alpha_{2}$ are restricted by $D_{1}, D_{2}$, and $S_{d}$. For instance, for $S_{d}$ given in the figure, $\alpha_{1}$ can not be 1 .

Lebacque suggested another supply-demand method: the supply of the downstream cell is first distributed to the two upstream cells with two fractions $\alpha_{1}$ and $\alpha_{2}$, and it is assumed that the flows $q_{1}$ and $q_{2}$ reaches their individual maximums. I.e., we can compute the flow $q_{i}(i=1,2)$ as the following:

$$
\begin{aligned}
S_{i} & =\alpha_{i} S_{d}, \\
q_{i} & =\min \left\{D_{i}, S_{i}\right\} .
\end{aligned}
$$


The feasible solutions of Lebacque's method without fixed fractions are shown in Figure 2. As shown, in this model, when $S_{d} \geq D_{1}+D_{2},\left(q_{1}, q_{2}\right)$ can be any point on $D_{1} B Q A D_{2} ;$ when $S_{d}<$ $D_{1}+D_{2},\left(q_{1}, q_{2}\right)$ can be any point on $D_{1} B A D_{2}$. In Lebacque's formulation, therefore, $\alpha_{1}$ and $\alpha_{2}$ are not restricted by $D_{1}, D_{2}$, or $S_{d}$, and the total flow $q$ may not reach its maximum $\min \left\{D_{1}+D_{2}, S_{d}\right\}$ in this method.

Comparing Daganzo's and Lebacque's model, we can see that: 1) when the fractions are the same, the two methods give the same flows; 2) for given $D_{1}, D_{2}$, and $S_{d}$, feasible solution domain of Daganzo's method is contained by that of Lebacque's since the distribution fractions in Daganzo's method (but not in Lebacque's) are confined by the supplies and the demand.

From the analysis above, we can see that both Daganzo's and Lebacque's models in the supplydemand framework are based on reasonable assumptions, and Lebacque's method (11) yields a larger set of feasible solutions than Daganzo's. In addition, we think that both formulations are clear and general enough to contain physical solutions. Thus, in this paper, we do not intend to investigate further on the formulations. Instead, we are interested in the distribution schemes used in these models.

The reasons why distribution scheme is worth further, deeper discussions are as follows. First, we can see from Figure 1 and Figure 2 that distribution schemes play a key role in uniquely determining flows through a merge. Thus whether solutions of flows are physical is highly dependent on the distribution scheme used. Therefore, in order to apply these models to simulate traffic dynamics at a merge, we need better understand their distribution schemes. Second, the distribution fractions can be affected by travellers' merging behaviors, the geometry of a merge, differences between the upstream cells, traffic conditions, and possible control strategies imposed on an on-ramp. On the surface, a distribution scheme that models all these factors should be 
extremely complicated. Thus we want to take at closer look at distribution schemes and hope that we can find a simple one. Third, it is possible that many valid distribution schemes are available. When this happens, a distribution scheme that is easy to calibrate and computationally efficient is always preferred. In this sense, further discussions are required to obtain such a scheme.

\section{Discussions on the distribution schemes}

In this section, we take a closer look at various distribution schemes and see how their distribution fractions are affected by traffic conditions, i.e., $D_{1}, D_{2}$, and $S_{d}$, and other characteristics of a merge. We start with review and discussion on the existing distribution schemes of Daganzo and Lebacque, and then propose a simple distribution scheme and demonstrate that the supply-demand method incorporating this scheme is capable of addressing all factors that we concern about.

\subsection{Discussions on existing distribution schemes}

As we know, different type of links have different characteristics. As a result, even when an upstream highway and an on-ramp have the same number of lanes and traffic density, the downstream link usually receives more vehicles from the upstream highway than from the on-ramp due to difference in design speed and geometry. For example, when vehicles queues up on both a $L$-lane highway and 1-lane on-ramp that merge together, the ratio of flow from the on-ramp to that from the highway is about 1/(2L-1) (Daganzo, 1996). From these observations, Daganzo (1995) assumed that different upstream links bear different priorities and suggested a distribution scheme including parameters for priorities.

Figure 3 shows how Daganzo's distribution scheme is defined. In the figure, the priorities of the highway and on-ramp are denoted as $p_{1}$ and $p_{2}\left(p_{1}+p_{2}=1\right)$ respectively. Here the upstream 
link $u_{1}$ is assumed to have higher priority than $u_{2}$; i.e., $p_{1} / p_{2}>D_{1} / D_{2}$. Then the solution $\left(q_{1}, q_{2}\right)$ can be shown in three cases: i) when $S_{d} \leq D_{1} / p_{1}$; i.e., $S_{d}$ is $x$ - and $y$-intercept of line i, the solution $\left(q_{1}, q_{2}\right)=\left(p_{1} S_{d}, p_{2} S_{d}\right)$ is at point 1 ; ii) when $S_{d} \in\left(D_{1} / p_{1}, D_{1}+D_{2}\right)$; i.e., $S_{d}$ is the $x$ - and $y$ intercept of line ii, the solution $\left(q_{1}, q_{2}\right)=\left(D_{1}, S_{d}-D_{1}\right)$ is at point 2 ; iii) when $S_{d} \geq D_{1}+D_{2}$; i.e., $S_{d}$ is the $x$ - and $y$ - intercept of line iii, the solution $\left(q_{1}, q_{2}\right)=\left(D_{1}, D_{2}\right)$ is at point 3 .

Thus in Daganzo's distribution scheme, we can find that the fraction $\alpha_{1}$ is defined as

$$
\alpha_{1}= \begin{cases}p_{1}, & S_{d} \leq D_{1} / p_{1} \\ \frac{D_{1}}{S_{d}}, & D_{1} / p_{1}<S_{d} \leq D_{1}+D_{2} \\ \frac{D_{1}}{D_{1}+D_{2}}, & S_{d}>D_{1}+D_{2}\end{cases}
$$

and $\alpha_{2}=1-\alpha_{1}$. From this definition and Figure 3 , we can see that the priorities $p_{1}$ and $p_{2}$ have to satisfy $p_{2} / p_{1}<D_{2} / D_{1}$. I.e., they have to change with respect to traffic conditions $D_{2}$ and $D_{1}$. Therefore, such "priorities" are not uniquely determined by road characteristics, as on would expect. This property makes this distribution scheme not so attractive. Moreover, even we allow non-constant priorities, the distribution scheme with fractions defined in (12) becomes quite complicated when considering a merge with more than two upstream links.

Lebacque (1996) suggested another distribution scheme, in which $\alpha_{i}$ equals to the ratio of the number of lanes of link $u_{i}$ to that of link $d$. When all branches of a merge are highways with the same characteristics, and the upstream links are overcritical, the demand of each upstream link is equal to capacity of a lane times the number of lanes. In this case, it is reasonable that outflow from each upstream link is proportional to the number of lanes; i.e., the distribution scheme by Lebacque works well. However, when the upstream links are not similar, e.g., one is highway and the other on-ramp, the fractions are obviously not proportional to the number of lanes. Lebacque's distribution scheme fails in another case when $\alpha_{1}+\alpha_{2}>1$ and $S_{i} \leq D_{i}$, because it may yield 
invalid solutions of $q=q_{1}+q_{2}>S_{d}$.

\subsection{A simple distribution scheme and its interpretation}

Our above analysis has revealed certain drawbacks of the existing distribution schemes, here we propose a simple distribution scheme, which, as we will see later, removes these drawbacks yet is capable of capturing characteristics of a merge. In this distribution scheme, the distribution fractions are only related to the demands $D_{1}$ and $D_{2}$, as defined in (13).

$$
\begin{aligned}
& \alpha_{1}=\frac{D_{1}}{D_{1}+D_{2}}, \\
& \alpha_{2}=\frac{D_{2}}{D_{1}+D_{2}} .
\end{aligned}
$$

As shown in Figure 4, combining the distribution scheme (13) with models (10) or (11), we are able to uniquely determine the flows: the solution $\left(q_{1}, q_{2}\right)$ with this distribution scheme is simply the intersection of $q_{1}+q_{2}=S_{d}$ and $q_{1} / q_{2}=D_{1} / D_{2}$ when $D_{1}+D_{2} \geq S_{d}$, and the point $Q$ otherwise.

A distribution scheme is in fact equivalent to an additional entropy condition, which helps identify $q_{1}$ and $q_{2}$. Thus we also call the distribution scheme (13) as the "fairness" condition, because the distribution fractions are proportional to traffic demands of upstream links; i.e., the upstream cell with more "sending" flow is given more chances. This "fairness" condition, to some extent, is supported by observations at crowded merges, e.g., vehicles from an on-ramp generally wait until there is a big enough gap to merge when traffic is fluid. When many vehicles from the on-ramp cannot merge and queue up, they may squeeze in and force vehicles from the upstream mainline freeway to slow down or switch lanes to give way to them (Kita, 1999). These observations show that vehicles from the upstream cells compete "fairly" with each other for admission into the downstream cell.

From (13), we can see that the distribution scheme is not directly related to capacities, number 
of lanes, the difference between upstream links, or control of on-ramps. Thus we can say that this distribution scheme uses the fewest parameters. Therefore, it will be easy to calibrate and efficient in computation. Indeed, it is the simplest distribution scheme that we can have. Besides, note that the fractions are independent of the downstream traffic conditions $S_{d}$, although the flows are related to $S_{d}$. As a mathematical excercise, the following theorem shows the fractions in this scheme are in fact the only fractions that are independent of $S_{d}$.

Theorem 1 Suppose that the fractions $\alpha_{1}$ and $\alpha_{2}$ are independent of the downstream supply $S_{d}$, then the fractions will be as in (13).

Proof. We have $q_{1}=\alpha_{1} q$ and $q_{2}=\alpha_{2} q$. From (11), we then obtain

$$
\begin{aligned}
& \alpha_{1} q \leq D_{1}, \\
& \alpha_{2} q \leq D_{2} .
\end{aligned}
$$

Since $\alpha_{1}$ and $\alpha_{2}$ are independent of $S_{d}$, set $S_{d}=D_{1}+D_{2}$, we obtain $q=D_{1}+D_{2}$. Thus, both (14) and (15) have to take the "=" sign, and we have (13).

On the other hand, characteristics of a merge can be captured in the simplest distribution scheme (13) since capacities, number of lanes, design speed, and control of on-ramp can be included in the definition of traffic demands, (6). For example, when upstream links have the same capacity per lane and are congested, (13) will give fractions proportional to number of lanes. Thus this distribution scheme addresses Lebacque's concern of number of lanes. As to Daganzo's concern, the "priorities" between the freeway and the on-ramp can be explained as follows: when the freeway and the onramp have the same number of lanes and density, the freeway generally admits higher free flow speed, has higher flow-rate and higher demand, and therefore has larger outflow. In addition, the resulted supply-demand method can be applied to determine flows through a merge with a 
controlled on-ramp (Daganzo, 1995): when the metering rate of the on-ramp, whose real traffic demand is $D_{2}$, is $r$, we can apply the controlled traffic demand of the on-ramp $\min \left\{r, D_{2}\right\}$ in the supply-demand method. Therefore, although the distribution scheme plays "fairly", the resulted supply-demand method of merges can address the characteristics of a merge by incorporating them into the computation of traffic demands and traffic supply.

From discussions above we can see that, in this simple distribution scheme, characteristics of upstream links, the control on on-ramps, and other properties of a merge are captured in the definitions of demand and supply. This is why distribution fractions can only be dependent on demands. Although demand functions of upstream cells are related to many factors and may not be easily obtained, they have to be found in all supply-demand methods. In this sense, the simple distribution scheme and, therefore, the supply-demand method with this scheme, are the easiest to calibrate and the most computationally efficient.

\subsection{Properties of the discrete kinematic wave model of merges with the simplest distribution scheme}

With the distribution scheme (13), the supply-demand method has the following further properties:

Equivalence of methods by Daganzo and Lebacque: With the distribution scheme (13), as shown in Figure 4, the solution $\left(q_{1}, q_{2}\right)$ will be on the line segment $O Q$. We can see that Daganzo's method (10) and Lebacque's method (11) are equivalent with these fractions. Thus, the supply-demand method can be rewritten as

$$
\begin{aligned}
q & =\min \left\{D_{1}+D_{2}, S_{d}\right\} \\
q_{1} & =q \cdot \frac{D_{1}}{D_{1}+D_{2}}, \\
q_{2} & =q \cdot \frac{D_{2}}{D_{1}+D_{2}} .
\end{aligned}
$$


Extensibility: The supply-demand method incorporating the simplest distribution scheme produces qualitatively similar results for merges with different number of upstream links. When a merge has $U>2$ upstream links, the method (16) can be easily extended as

$$
\begin{aligned}
q & =\min \left\{\sum_{i=1}^{U} D_{i}, S_{d}\right\}, \\
q_{i} & =q \frac{D_{i}}{\sum_{i=1}^{U} D_{i}}, \quad i=1, \cdots, U .
\end{aligned}
$$

Convergence of the merge model: The discrete LWR model is considered as a good approximation to the continuous LWR model since it converges as $\Delta x \rightarrow 0$ while $\Delta x / \Delta t$ is constant; although the analytical analysis of convergence of the merge model with the simplest distribution scheme has not yet been done, numerical results in section 4 will show that it is convergent.

Consistency of the merge model with the LWR model: Here we conceptually consider the consistency of the merge model (16) with the LWR model for a link with multiple lanes. In the LWR model for a multi-lane link, all lanes are assumed to be identical; i.e., given the same initial and boundary conditions for each lane, flows at the same location on each lane are identical and the link's flow-rate or density at a location is simply the number of lanes times the flow-rate or density at the location of each lane, respectively. I.e., the LWR model does not capture the lane-changing effects of vehicles and lanes are effectively isolated. Therefore a multi-lane link can be considered as an equivalent merge: for a boundary inside the link, we separate its upstream part into two links, while keep the downstream part intact, and still assume that the two artificial upstream links have identical lanes. Next we will check traffic dynamics of this artificial merge are indeed the same as those of the link. Assuming the two upstream links of an artificial merge, which is constructed from a boundary, have $N$ and $M$ lanes respectively, traffic demand of each lane is $D$, and traffic supply of the downstream link 
is $S_{d}$. Since the lanes are identical in the upstream links, traffic demands for the links are $D_{1}=N D, D_{2}=M D ;$ from (16), we have

$$
\begin{aligned}
q & =\min \left\{(N+M) D, S_{d}\right\} \\
q_{1} & =q \frac{N}{N+M} \\
q_{2} & =q \frac{M}{N+M} .
\end{aligned}
$$

Hence, as expected, flow from each upstream lane is $\min \left\{D, S_{d} /(N+M)\right\}$, which is the same as the original boundary flow computed from the LWR model.

From the above analysis, we can see that the supply-demand merge model with the simplest distribution scheme is well-defined.

\section{Numerical simulations}

In this section, we present our numerical studies of the discrete kinematic wave model of merges using the simple distribution scheme. Here we apply Godunov's method discussed in Subsection 2.1 for each link, and the supply-demand method is used to find flows through link boundaries and merging boundaries. Especially, the simple distribution scheme is used for computing fluxes through the merge. The resulted numerical solution method is described as follows: in each cell, (4) is used to update traffic density; we compute flows through link boundaries with (9); flows through a merge are computed from (17).

In the numerical studies, we introduce a unit time $\tau=5 \mathrm{~s}$ and a unit length $l=0.028 \mathrm{~km}$. Here we study a merge formed by a two-lane mainline freeway and a one-lane on-ramp. The three branches of the merge have the same length, $L=400 l=11.2 \mathrm{~km}$, and the upstream mainline freeway, the on-ramp, and the downstream mainline freeway are labeled as links $u_{1}$, $u_{2}$, and $d$, 
respectively. The simulation starts from $t=0$ and ends at $t=500 \tau=41.7 \mathrm{~min}$. In the following numerical simulations, we partition each link into $N$ cells and the time interval into $K$ steps, with $N / K=1 / 10$ always; e.g., if $N=50$ and $K=500$, the cell length is $\Delta x=8 l$ and the length of each time step $\Delta t=1 \tau$.

For both the mainline freeway and the on-ramp, we use the triangular fundamental diagram; i.e., the flow-density relationships are triangular. For the mainline freeway, the free flow speed is $v_{f, m}=65 \mathrm{mph}=5.1877 \mathrm{l} / \tau$; the jam density is $\rho_{j, m}=2 \rho_{j}=360 \mathrm{veh} / \mathrm{km}$, where $\rho_{j}=180 \mathrm{veh} / \mathrm{km} /$ lane is the jam density of a single lane; and the critical density $\rho_{c, m}=0.2 \rho_{j, m}=0.4 \rho_{j}=72 \mathrm{veh} / \mathrm{km}$. Therefore, the speed- and flow-density relationships can be written as follows:

$$
\begin{aligned}
& V_{m}(\rho)= \begin{cases}v_{f, m}, & 0 \leq \rho \leq \rho_{c, m} ; \\
\frac{\rho_{c, m}}{\rho_{j, m}-\rho_{c, m}} \frac{\rho_{j, m}-\rho}{\rho} v_{f, m}, & \rho_{c, m}<\rho \leq \rho_{j, m} .\end{cases} \\
& Q_{m}(\rho)=\rho V_{m}(\rho)= \begin{cases}v_{f, m} \rho, & 0 \leq \rho \leq \rho_{c, m} ; \\
\frac{\rho_{c, m}}{\rho_{j, m}-\rho_{c, m}} v_{f, m}\left(\rho_{j, m}-\rho\right), & \rho_{c, m}<\rho \leq \rho_{j, m} .\end{cases}
\end{aligned}
$$

For the on-ramp, the free flow speed $v_{f, r}=35 \mathrm{mph}=2.7934 \mathrm{l} / \tau$; the jam density is $\rho_{j}$; and the critical density $\rho_{c, r}=0.2 \rho_{j}$. Similarly, we can have the following speed- and flow-density relationships:

$$
\begin{aligned}
& V_{r}(\rho)= \begin{cases}v_{f, r}, & 0 \leq \rho \leq \rho_{c, r} ; \\
\frac{\rho_{c, r}}{\rho_{j}-\rho_{c, r}} \frac{\rho_{j}-\rho}{\rho} v_{f, r}, & \rho_{c, r}<\rho \leq \rho_{j} .\end{cases} \\
& Q_{r}(\rho)=\rho V_{r}(\rho)= \begin{cases}v_{f, r} \rho, & 0 \leq \rho \leq \rho_{c, r} ; \\
\frac{\rho_{c, r}}{\rho_{j}-\rho_{c, r}} v_{f, r}\left(\rho_{j}-\rho\right), & \rho_{c, r}<\rho \leq \rho_{j} .\end{cases}
\end{aligned}
$$

The above relationships are depicted in Figure 5.

Since $\left|\lambda_{*, m}\right| \leq v_{f, m}=5.1877 l / \tau$, where $\lambda_{*, m}(\rho)=V_{m}(\rho)+\rho V_{m}^{\prime}(\rho)$ is the characteristic speed of (3) on the mainline freeway, we find the CFL (Courant, Friedrichs and Lewy, 1928) condition 
number

$$
\left|\lambda_{*, m}\right| \frac{\Delta t}{\Delta x} \leq 0.65<1
$$

Since the characteristic speed on the on-ramp is smaller than that on the mainline freeway, the CFL condition is also satisfied for the on-ramp. Therefore, the first-order Godunov's method can solve the LWR model efficiently.

\subsection{Simulation of merging traffic without control}

In this subsection, we study the following merging traffic. Initially, the mainline freeway carries a constant flow, which is under-critical: traffic densities on the upstream and downstream freeway are the same, $\rho_{u_{1}}=\rho_{u_{2}}=0.36 \rho_{j}$. After time $t=0$, a constant flow $\rho_{u_{2}}=0.175 \rho_{j}$ is introduced by the on-ramp, and the on-ramp remains uncontrolled. In our simulation, the Riemann boundary condition is imposed for the upstream boundaries of link $u_{1}$ and $u_{2}$ and the downstream boundary of link $d$; i.e. the derivatives of traffic density at these boundaries are assumed to be zero.

After partitioning each of the three links into $N=500$ cells and discretizing the time duration $[0,500 \tau]$ into $K=5000$ steps, we obtain simulation results as shown in Figure 6 . Among these figures, Figure (a) illustrates the evolution of traffic density on the upstream freeway: at time $t=0 \tau$, traffic density is uniformly $\rho_{A}=0.36 \rho_{j}$; after the introduction of on-ramp flow, traffic at the merging point becomes congested and reaches a new state $\rho_{B}=0.7394 \rho_{j}$; then a shock wave forms on the upstream mainline freeway since the downstream traffic has higher density, and the shock wave travels upstream in a constant speed $s_{1} \approx-0.61 l \tau=-7.6 \mathrm{mph}$. Figure $(\mathrm{b})$ shows the evolution of traffic on the downstream mainline highway: initially, traffic density is also uniformly $\rho_{A}$; after $t=0$, traffic at the merging point reaches the capacity flow $\rho_{C}=0.4 \rho_{j}$, and a rarefaction wave appears since $\rho_{C}>\rho_{A}$; however, due to the special structure of the triangular 
fundamental diagram, the rarefaction wave is similar to a shock wave and travels downstream in the speed $s_{2} \approx 5.2 l / \tau=v_{f, m}$. Figure (c) demonstrates that a backward shock wave forms on the on-ramp: the upstream of the shock wave has density $\rho_{D}=0.175 \rho_{j}$ and the downstream has density $\rho_{E}=0.3697 \rho_{j}$, and the shock wave speed is $s_{3} \approx-0.25 l / \tau=-3.1 \mathrm{mph}$. The shock waves and rarefaction wave observed on the three branches in the $\rho-q$ phase plane are shown in Figure (d), in which line $A B$ represents the shock wave on the upstream mainline freeway and the slope of $A B$ is equal to the shock wave speed, line $C A$ represents the rarefaction wave on the downstream mainline freeway and the slope of $C A$ is equal to the free flow speed and the rarefaction wave speed, and line $D E$ represents the shock wave on the on-ramp and the slope of $D E$ is equal to the shock wave speed.

Comparing the congested state on the upstream freeway (point $B$ in (d) of Figure 6) and that on the on-ramp (point $E$ in (d) of Figure 6 ), we have the following results. First, $\rho_{E}=\frac{1}{2} \rho_{B}$, i.e., at the merging point traffic density is equal on all upstream lanes, whether they are on the freeway or the on-ramp. This is because all lanes have the same jam density and critical density and the onramp is not controlled. Second, the outflow from the upstream freeway is $q_{B}=1.6349 \rho_{j} l / \tau=5933$ veh/hr, and the outflow from the on-ramp is $q_{E}=0.4402 \rho_{j} l / \tau=1597 \mathrm{veh} / \mathrm{hr}$. Since $q_{E} \neq \frac{1}{2} q_{B}$, the outflows from the freeway and on-ramp are not proportional to the number of lanes. As analyzed before, this is due to the difference between the free flow speeds. Third, we expect that $\rho_{B}, \rho_{E}$, and $\rho_{C}$ are always the same even with different initial traffic conditions, given that the downstream freeway is not over-critical, which is equivalent to saying that its supply is the capacity, and that the total demand of the upstream branches is larger than the capacity of the freeway. 


\subsection{Simulation of merging traffic when the on-ramp is controlled}

In this subsection, we adopt exactly the same assumptions as in the preceding subsection, except that the on-ramp is controlled with a metering scheme. Here the metering rate $r=0.3445 \rho_{j} l / \tau=1250$ veh/hr. The simulation results are given in Figure 7. From Figure (a), we can see that a backward traveling shock wave forms on the upstream freeway, the upstream of the shock wave has the initial density $\rho_{A}=0.36 \rho_{j}$, the downstream of the shock wave has a larger density $\rho_{B}=0.6278 \rho_{j}$, and the shock wave speed is $s_{1} \approx-0.33 l / \tau=-4.1 \mathrm{mph}$. Comparing this figure with (a) of Figure 6, we find that, with on-ramp metering, the upstream highway becomes less congested and the absolute value of the shock wave speed is smaller. Figure (b) shows that the evolution of traffic on the downstream freeway is the same as in the previous simulation without on-ramp control. From Figure (c), we can see that a backward traveling shock wave forms on the on-ramp, traffic densities on the upstream and downstream parts of the shock wave are the initial density $\rho_{D}=0.175 \rho_{j}$ and $\rho_{E}=0.577 \rho_{j}$ respectively, and the shock wave speed is $s_{3} \approx-0.48 l / \tau=-6.0 \mathrm{mph}$. Thus, the on-ramp becomes more congested and the congested area prevails faster with on-ramp metering. Figure (d) presents the wave solutions in the $\rho-q$ plane.

Comparing the congested state on the upstream freeway (point $B$ in (d) of Figure 6) and that on the on-ramp (point $E$ in (d) of Figure 6, we also have different results from those in the

previous simulation. First, $\rho_{E}>\frac{1}{2} \rho_{B}$, which means traffic on the on-ramp is much denser. Second, $q_{B}=1.7797 \rho_{j} l / \tau=6458 \mathrm{veh} / \mathrm{hr}$ and $q_{E}=0.2954 \rho_{j} l / \tau=1072 \mathrm{veh} / \mathrm{hr}$. Thus the outflow from the upstream freeway is higher than without control and the outflow from the on-ramp is lower. Here the outflow from the on-ramp, $q_{E}$, is also lower than the metering rate $r$, since $r$ gives the upper bound of traffic demand. Third, the on-ramp control changes the distribution fractions: without control, $\alpha_{1}=q_{B} /\left(q_{B}+q_{E}\right)=78.8 \%$; with control, $\alpha_{1}=85.8 \%$. 
The above results conform to our expectation that the on-ramp control can improve traffic conditions on the mainline freeway by restricting the access of vehicles from the on-ramp.

\subsection{Computation of convergence rates}

In this subsection we will check the convergence of the merge model with the distribution scheme (13), when the on-ramp is not controlled. Here, this is done by computing convergence rates of traffic density over the whole network.

First, we compare traffic density solutions at time $T_{0}=500 \tau$ for two different number of cells into which the network is partitioned and obtain their difference. Denote solutions as $\left(U_{i}^{2 N}\right)_{i=1}^{2 N}$ for

$2 N$ cells and $\left(U_{i}^{N}\right)_{i=1}^{N}$ for $N$ cells respectively, and define a difference vector $\left(e^{2 N-N}\right)_{i=1}^{N}$ between these two solutions as

$$
\mathbf{e}_{i}^{2 N-N}=\frac{1}{2}\left(U_{2 i-1}^{2 N}+U_{2 i}^{2 N}\right)-U_{i}^{N}, i=1, \cdots, N
$$

Then, the relative error with respect to $L^{1}-, L^{2}$ - or $L^{\infty}$-norm can be computed as

$$
\epsilon^{2 N-N}=\left\|\mathbf{e}^{2 N-N}\right\|
$$

Finally, a convergence rate is obtained when we compare two relative errors:

$$
r=\log _{2}\left(\frac{\epsilon^{2 N-N}}{\epsilon^{4 N-2 N}}\right)
$$

Here the vector of $U$ contains the densities of all three links which are weighted by the number of lanes of each link.

We will compute convergence rate with the following conditions. For link $u_{1}$, the number of lanes $a\left(u_{1}\right)=2$, we define its initial condition as

$$
\begin{array}{ll}
\rho(x, 0)=a\left(u_{1}\right)\left(0.18+0.05 \sin \frac{\pi x}{L}\right) \rho_{j}, & x \in[0, L], \\
v(x, 0)=V_{m}(\rho(x, 0)), & x \in[0, L] .
\end{array}
$$




\begin{tabular}{lccccccc}
\hline$\rho / \rho_{j}$ & $128-64$ & Rate & $256-128$ & Rate & $512-256$ & Rate & $1024-512$ \\
\hline$L^{1}$ & $3.31 \mathrm{e}-03$ & 1.00 & $1.65 \mathrm{e}-03$ & 1.00 & $8.27 \mathrm{e}-04$ & 1.00 & $4.13 \mathrm{e}-04$ \\
\hline$L^{2}$ & $2.17 \mathrm{e}-02$ & 0.53 & $1.50 \mathrm{e}-02$ & 0.50 & $1.06 \mathrm{e}-02$ & 0.50 & $7.49 \mathrm{e}-03$ \\
\hline$L^{\infty}$ & $1.62 \mathrm{e}-01$ & 0.23 & $1.39 \mathrm{e}-01$ & 0.07 & $1.32 \mathrm{e}-01$ & 0.01 & $1.30 \mathrm{e}-01$ \\
\hline \hline
\end{tabular}

Table 1: Convergence rates of the discrete merge model

For link $u_{2}$, the number of lanes $a\left(u_{2}\right)=1$, we define its initial condition as

$$
\begin{array}{lll}
\rho(x, 0)=a\left(u_{2}\right)\left(0.175+0.05 \sin \frac{2 \pi x}{L}\right) \rho_{j}, & & x \in[0, L], \\
v(x, 0)=V_{r}(\rho(x, 0)), & & x \in[0, L] .
\end{array}
$$

For link $d$, the number of lanes $a(d)=2$, we define its initial condition as

$$
\begin{array}{ll}
\rho(x, 0)=a(d)\left(0.18-0.05 \sin \frac{\pi x}{L}\right) \rho_{j}, & x \in[L, 2 L], \\
v(x, 0)=V_{m}(\rho(x, 0)), & x \in[L, 2 L] .
\end{array}
$$

In addition, we impose the Riemann boundary condition for the upstream boundaries of link $u_{1}$ and $u_{2}$ and the downstream boundary of link $d$; i.e., $\rho$ has zero derivative at these boundaries.

From Table 1, convergence rates of the merge model with the simple distribution scheme are of order one in $L^{1}$ norm. That the convergence rates in $L^{2}$ and $L^{\infty}$ norms are much smaller is believed to be caused by the special structure of the triangular fundamental diagram. However, it can still be seen that the discrete merge model is convergent with given initial conditions. 


\section{Discussions}

In this paper, we studied the discrete kinematic wave model of merges with the supply-demand method, probed the supply-demand models by Daganzo and Lebacque, and gained a better understanding of various distribution schemes. More important, we proposed the simplest distribution scheme, equivalent to the so-called "fairness" condition, in which the distribution fractions are proportional to the upstream demands. We demonstrated with both analytical discussions and numerical studies that the discrete merge model with the simplest distribution scheme is well-defined.

The simple distribution scheme can also be easily extended to diverges in single-commodity traffic flow. In reality, a diverge is not simply a reverse of a merge: in a merge, vehicles advance in the same direction and once they travel into the downstream link, their origins no longer affect traffic dynamics; while in a diverge, traffic dynamics in the upstream link are affected by the combination of vehicles with different destinations. However, for single-commodity traffic flows, in which vehicles have no predefined routes and can choose any downstream links when diverging, we can have similar supply-demand method for computing fluxes through a diverge: assuming that a diverge has $K$ downstream links, the upstream link and the downstream links are properly discretized, the traffic demand of the upstream link is $D_{u}$, and traffic supply of downstream link $d_{i}$ $(i=1, \cdots, K)$ is $S_{i}$, the supply-demand method with the "fairness" condition, in which the influx into a downstream link is proportional to its accommodation $S_{i}$, can be written as:

$$
\begin{aligned}
q & =\min \left\{D_{u}, \sum_{i}^{K} S_{i}\right\}, \\
q_{i} & =p_{i} q, \\
p_{i} & =\frac{S_{i}}{\sum_{i}^{K} S_{i}}, \quad i=1, \cdots, K,
\end{aligned}
$$

where $q_{i}$ is influx into the downstream link $d_{i}$.

Further, for a general junction with $J$ upstream links and $K$ downstream links, we can compute 
fluxes as

$$
\begin{aligned}
q & =\min \left\{\sum_{j=1}^{J} D_{j}, \sum_{k=1}^{K} S_{k}\right\}, \\
q_{j}^{u} & =\alpha_{j} q, \\
q_{k}^{d} & =\beta_{k} q, \\
\alpha_{j} & =\frac{D_{j}}{\sum_{j=1}^{J} D_{j}}, \quad j=1, \cdots, J \\
\beta_{k} & =\frac{S_{k}}{\sum_{k=1}^{K} S_{k}}, \quad k=1, \cdots, K,
\end{aligned}
$$

where $D_{j}$ is the traffic demand of the upstream link $u_{j}, S_{k}$ is the traffic supply of the downstream

link $d_{k}, q_{j}^{u}$ is the out-flux from $u_{j}$, and $q_{k}^{d}$ the influx into $d_{k}$. Although this model may not be proper for realistic traffic flow, it could be helpful for the study of other unidirectional flows.

With a complete picture of the merge model, we can now simulate how a traffic disturbance propagates through a merge. Especially, since merges are well recognized as locations where congestions always initiate (Daganzo, 1999), this study will help traffic engineers understand how congestions start and propagate at a merge. Moreover, with the complete discrete kinematic wave merge model, we are able to develop and evaluate local ramp metering strategies, e.g., the ALINEA algorithm (Papageorgiou et al., 1997). In the future, we will be also interested in the limit of the discrete kinematic wave model with the simple distribution scheme. Especially, we will be interested in kinematic wave solutions for a general Riemann problem. Finally, and more importantly, the supply-demand method and the "fairness" condition needs to be checked against observed merging phenomena.

\section{Acknowledgment}

This research is supported in part by the National Science Foundation through a CAREER research grant. 


\section{References}

O. Chen, A. Hotz, and M. Ben-Akiva. Development and evaluation of a dynamic metering control model. In IFAC Transportation Systems, Chania, Greece, 1997.

R. Courant, K. Friedrichs, and H. Lewy. ber die partiellen differenzengleichungen der mathematischen physik. Math. Ann., 100:32-74, 1928.

Carlos F. Daganzo. The cell transmission model part ii: Network traffic. Transportation Research Part B, 29(2):79-93, 1995.

Carlos F. Daganzo. The nature of freeway gridlock and how to prevent it. In The International Symposium on Transportation and Traffic Theory, Lyon, France, 1996.

Carlos F. Daganzo. Remarks on traffic flow modeling and its applications. In Proc. Traffic and Mobility - Simulation, Acachen, Germany, 1999. Economics and Environement Conference. in press.

S.K. Godunov. A difference method for numerical calculations of discontinuous solutions of the equations of hydrodynamics. Mat. Sb., 47:271-306, 1959. In Russian.

H. Holden and N.H. Risebro. A mathematical model of traffic flow on a network of unidirectional roads. SIAM J. MATH. ANAL., 26(4):999-1017, 1995.

E.I. Isaacson and J.B. Temple. Nonlinear resonance in systems of conservation laws. SIAM J. Appl. Math., 52(5):1260-1278, 1992.

W.L. Jin and H.M. Zhang. The inhomogeneous kinematic wave traffic flow model as a resonant nonlinear system. 2000. submitted. 
B.S. Kerner and P. Konhäuser. Structure and parameters of clusters in traffic flow. Physical Review E, 50(1):54-83, 1994.

H. Kita. A merging-giveaway interaction model of cars in a merging section : a game theoretic analysis. Transportation Research Part A, 33(3):305-312, 1999.

P.D. Lax. Hyperbolic systems of conservation laws and the mathematical theory of shock waves. SIAM, Philadelphia, Pennsylvania, 1972.

J.P. Lebacque. The godunov scheme and what it means for first order traffic flow models. In The International Symposium on Transportation and Traffic Theory, Lyon, France, 1996.

M.J. Lighthill and G.B. Whitham. On kinematic waves: Ii. a theory of traffic flow on long crowded roads. Proc. Royal Society A, 229:317-345, 1955.

H. Lo. A dynamic traffic assignment formulation that encapsulates the cell transmission model. In A. Cedar, editor, Transportation and Traffic Theory, pages 327-350. Elsevier Science, 1999.

G. F. Newell. A simplified theory of kinematic waves in highway traffic i: General theory. ii: Queuing at freeway bottlenecks. iii: Multi-destination flows. Transportation Research Part B, 27:281-313, 1993.

M. Papageorgiou, H. Hadj-Salem, and F. Middelham. Alinea local ramp metering - summary of field results. Transportation Research Record 1603, 1997.

P.I. Richards. Shock waves on the highway. Operations Research, 4:42-51, 1956. 


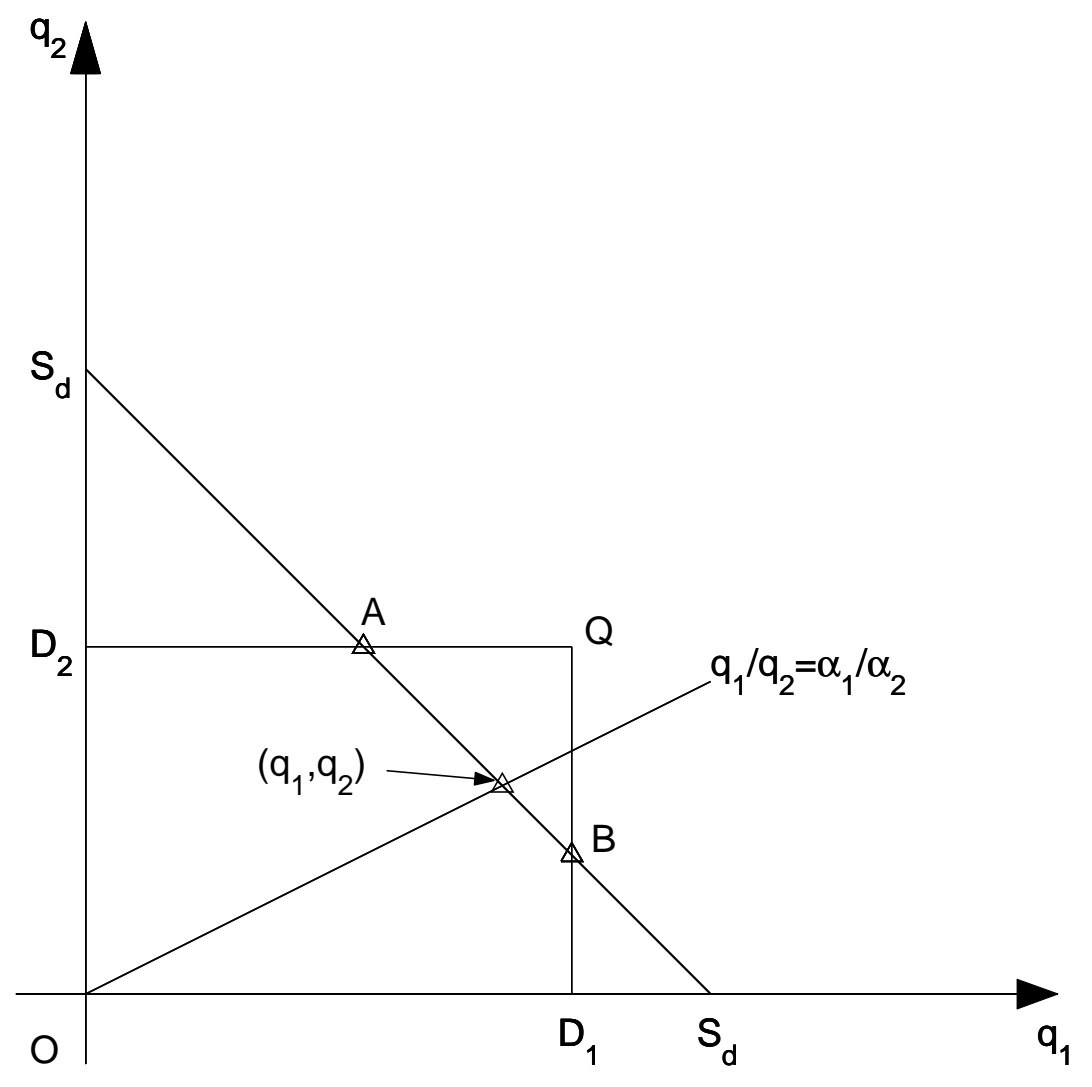

Figure 1: Feasible solutions in Daganzo's supply-demand method 


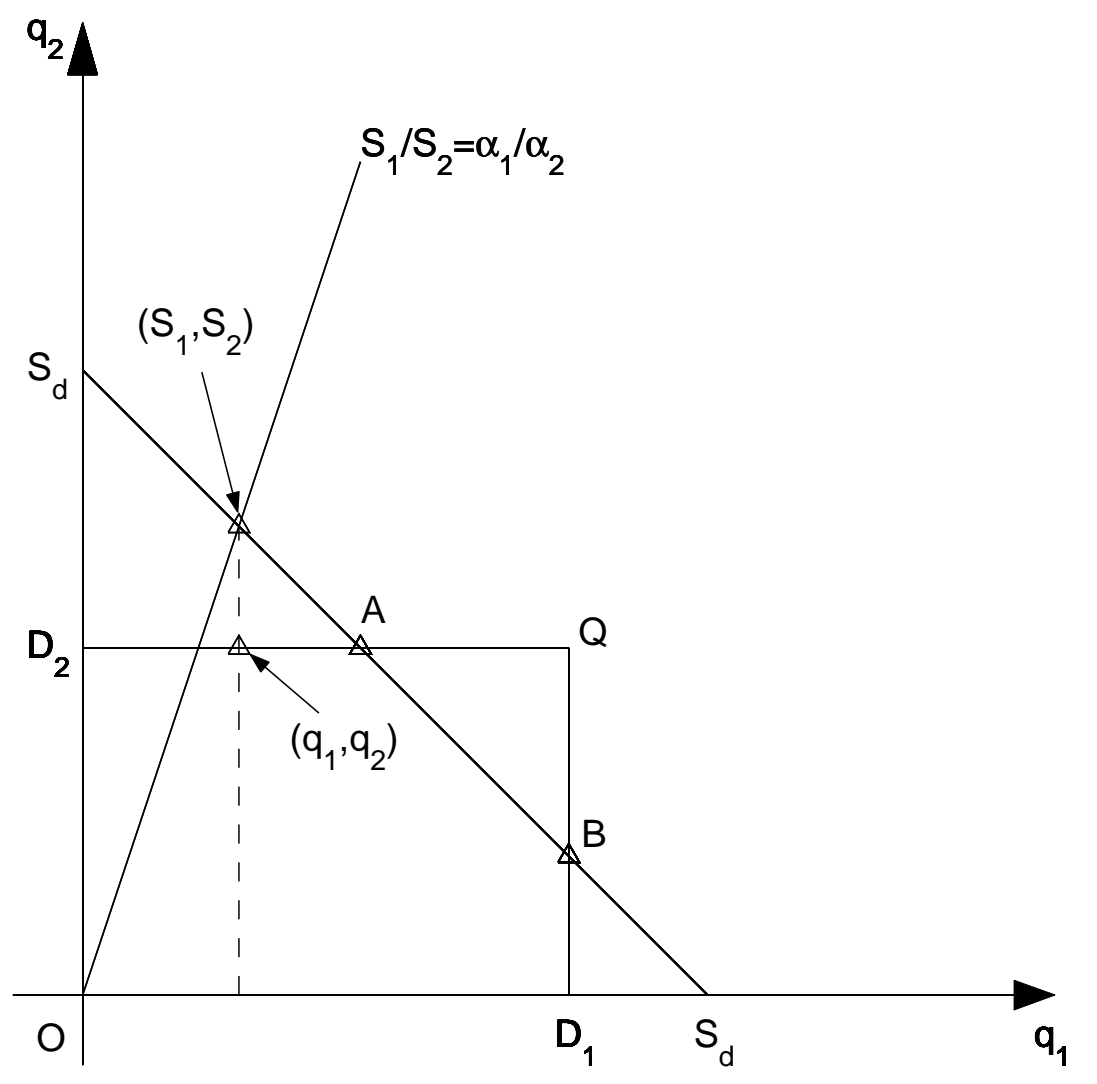

Figure 2: Feasible solutions in Lebacque's supply-demand method 


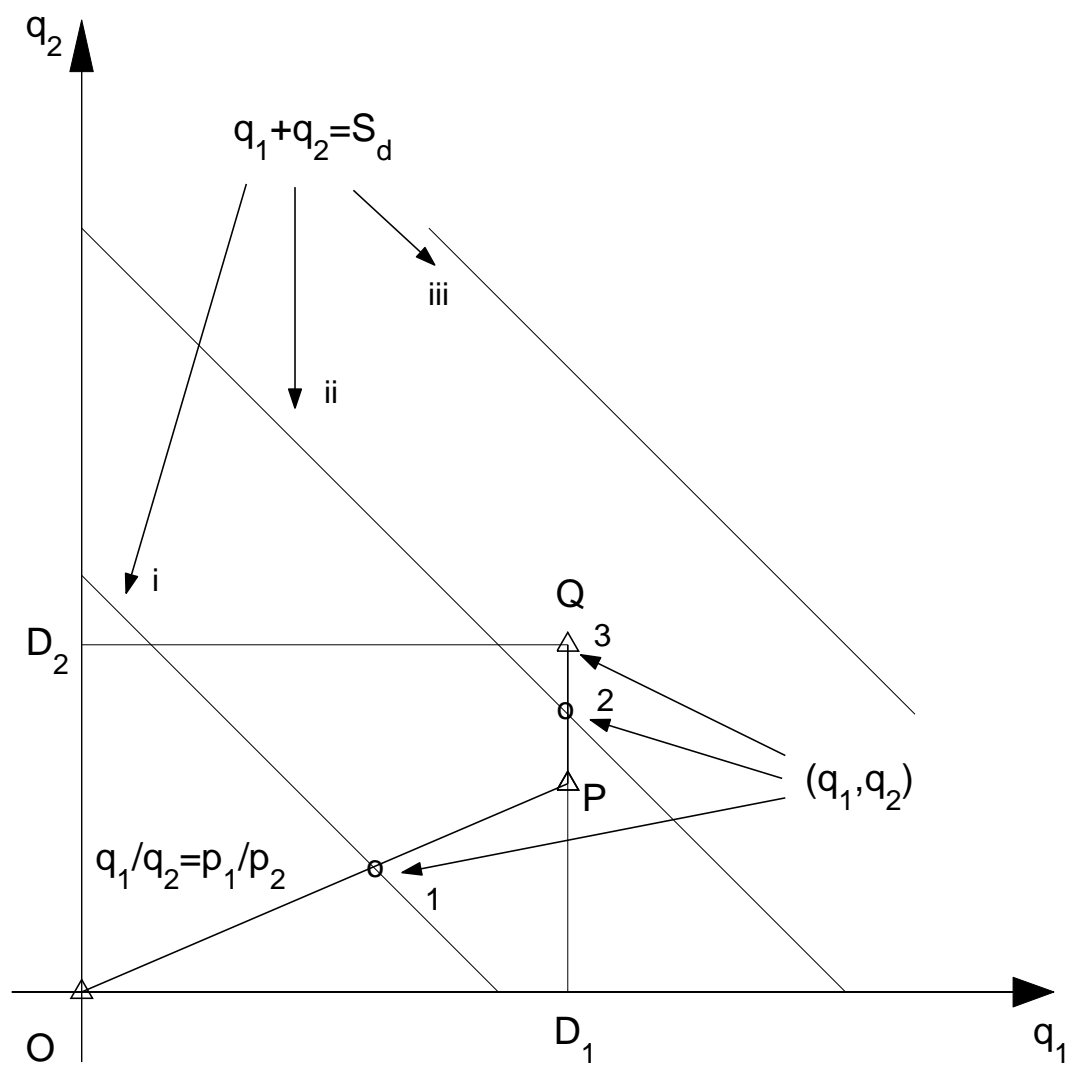

Figure 3: Solutions of flows in Daganzo's distribution scheme 


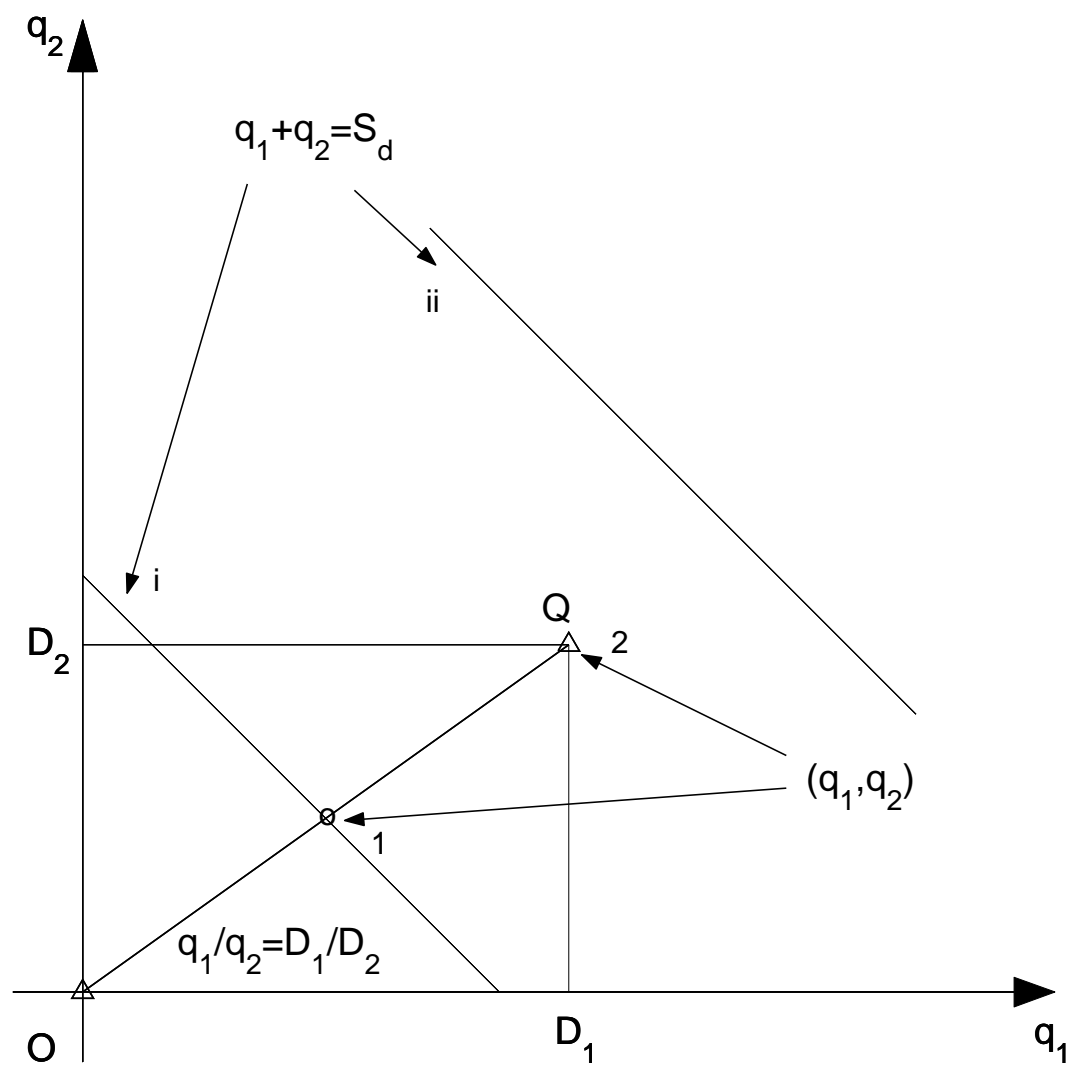

Figure 4: Solutions of flows in the simple distribution scheme 

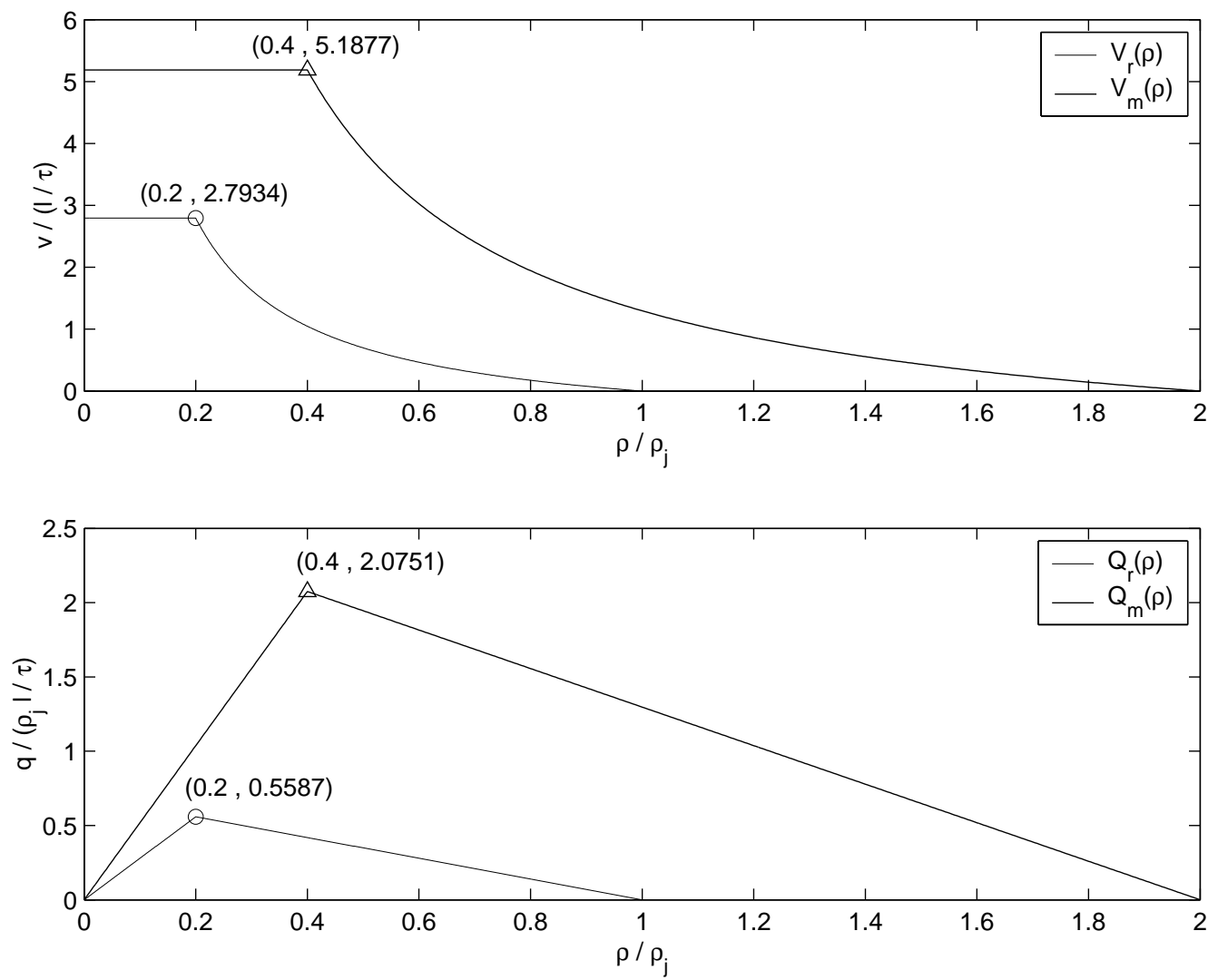

Figure 5: The triangular fundamental diagrams for the mainline freeway and the on-ramp 
(a)

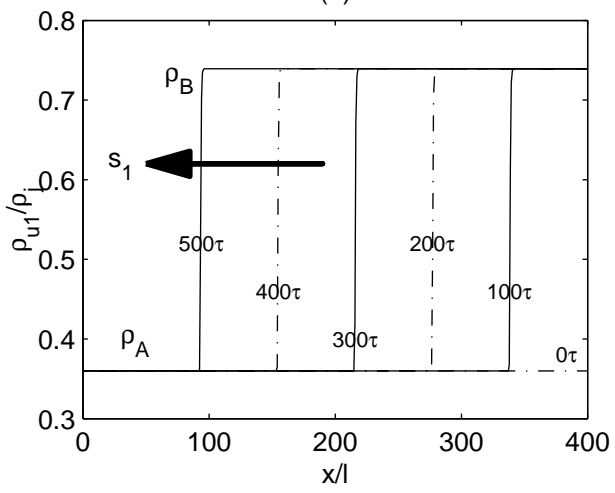

(c)

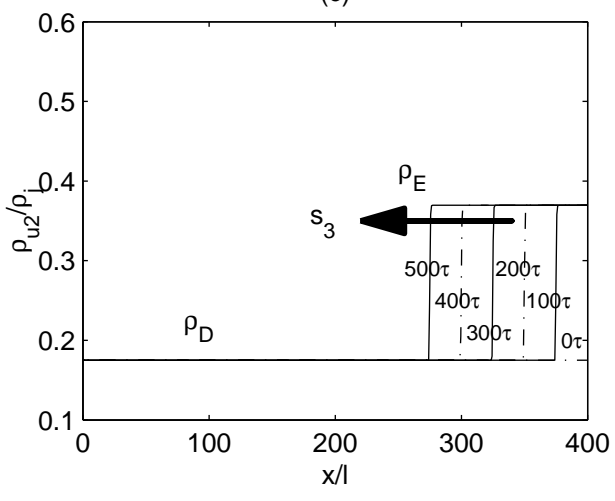

(b)

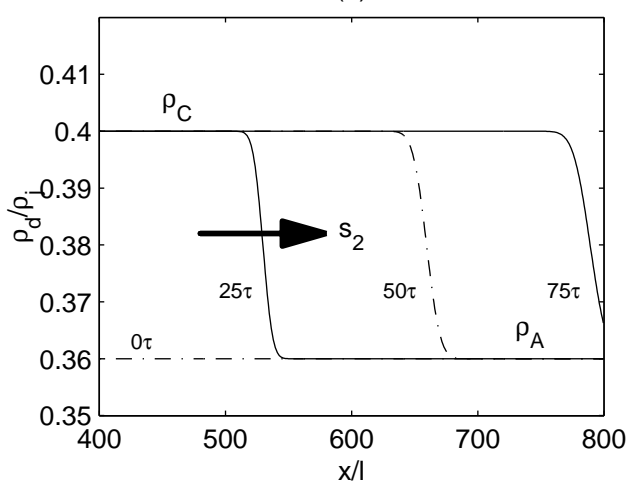

(d)

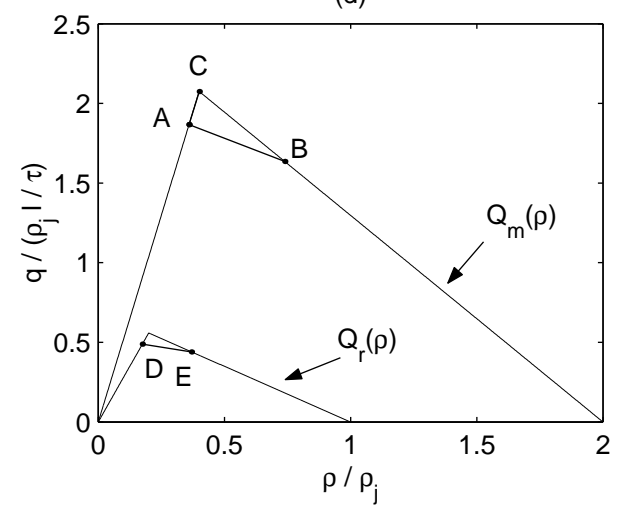

Figure 6: Simulation of merging traffic without control 
(a)

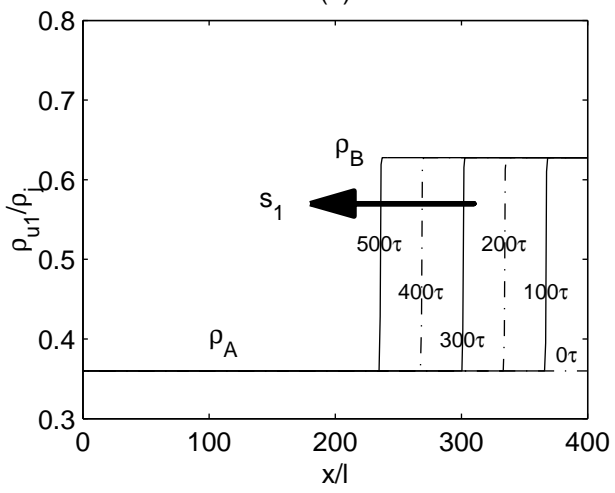

(c)

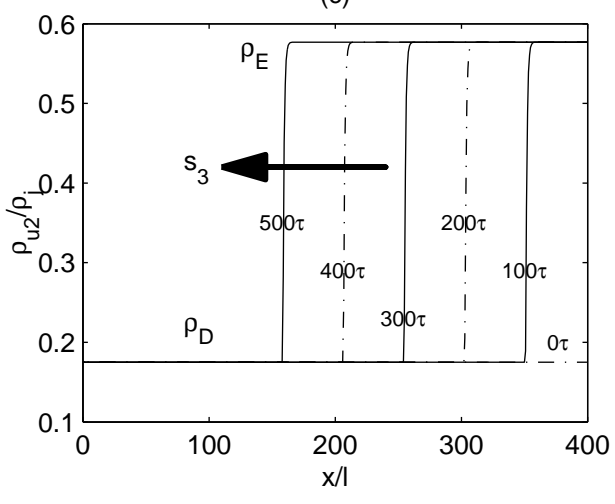

(b)

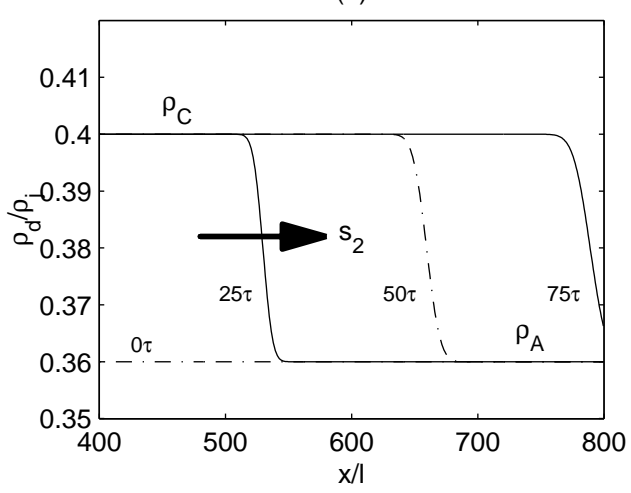

(d)

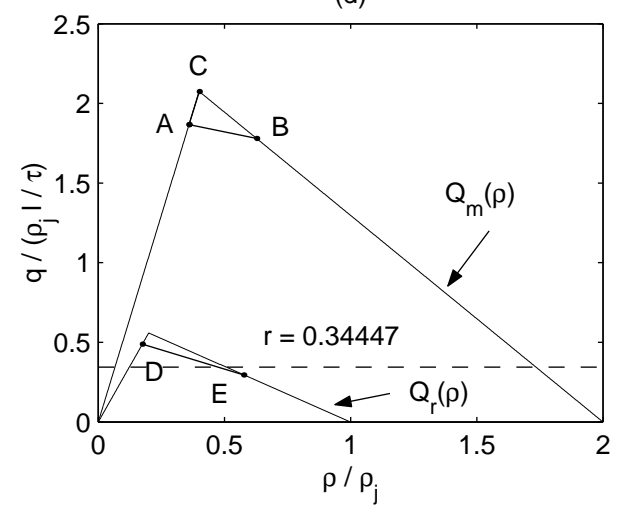

Figure 7: Simulation of merging traffic with on-ramp control 